\title{
Cognitive Process Observation Based on Human-Computer Interaction
}

\author{
Cheng Rengui ${ }^{1}$, Liu Changyong ${ }^{2}$, Yu Wensen ${ }^{1}$, Guo Lei $^{1}$ and Meng Shimin ${ }^{1,3, *}$ \\ ${ }^{1}$ School of Mathematics and Computer, Wuyi University, Wuyishan, Fujian, 354300, China \\ ${ }^{2}$ School of Modern Information Service, Wuyi University, Wuyishan, Fujian, 354300, China \\ ${ }^{3}$ Brain cognitive structure mapping Lab @ UlinkM.com INC., Wuyishan, Fujian, 354300, China
}

\begin{abstract}
Brain research focuses on matter, energy and information. Brain imaging of cognitive function concentrates on detecting brain activity in the energy layer and is the mainstream research paradigm in current brain science, hotspot patents. However, the core function of the brain is to process and dissipate information. It is extremely difficult to deduce brain activity and information processing mechanism based on activities in the energy layer; Studying the information processing mechanism of the brain directly from the information and content layers is a direction worth exploring. In terms of psychology, the basic object of brain research in the content layer is the cognitive structure. The cognitive structure is the basis for cognition, learning and thinking, so it is undoubtedly objective. Directly collecting cognitive information and reconstructing the methods for observing cognitive structures are scientific problems that deserve to be studied. How to observe the cognitive structure inside the brain? The new cognitive view holds that cognition occurs in the coupling interaction between the brain and the environment and the innovative method for observing the cognitive structure of man-machine coupling views the environment for man-machine interaction as the digital platform for cognition observation consisting of the brain cognitive body, the coupling situation and the information manifold (BSM). BSM is a combination of information and cognition. It should be analyzed from the perspectives of mathematic foundation, observation methods, imaging technology and cognitive dynamics. Cognitive structure imaging will be the new direction of the brain technology patents.
\end{abstract}

Keywords: Agent, cognitive structures, complete network, manifold, visualization.

\section{INTRODUCTION}

The important goals of information science in this century is to observe the cognitive activity, the information processing mechanism and the intelligence evolution of the brain, to collect cognitive data to construct cognitive structure models, to study the systematic law of cognitive dynamics and to explore the secrets of brain cognition. Compared with the vast universe and tiny atoms, human study on brain cognition is still in its infancy [1-3]. ERWIN SCHRODINGER thinking: Any description of the changes in the nervous system, and certainly does not contain "yellow", "sweet" characteristic explanation as to the objective description of the electromagnetic wave does not contain these characteristics as explained [4]. It is irreplaceable that cognitive content of brain research. What has hindered our study on the universe inside the brain? In our opinion, it is essential to innovate research paradigms, tools, and mathematical methods and especially to construct such tools for detecting cognitive structures such as telescopes, microscopes and particle accelerators.

\subsection{Simulation of the Cognitive Function of Man- Machine Separation}

In the current brain research paradigms, it is generally considered that the psychological mechanism of the information processing of the human brain can neither directly detect the implicit psychological mechanism from the outside nor directly observe the psychological processes of human brain tissue at the microscopic level, therefore, Computer Aided Methods or the so-called "Black Box" method are used with the help of theoretical thinking that indirectly deduces the internal activities and mechanisms of study objects. Above is the implicit hypothesis of brain research in the contemporary cognition science which we call the research paradigm "the functional simulation of man-machine separation". Functional simulation cannot directly collect the data of brain cognitive activity, nor is it able to build a model for quantifying cognitive dynamics based on data.

According to the new cognitive perspective, cognition occurs in the coupling interaction between the brain and the environment, while man-machine interaction is a close combination of the brain and the computer. Can we view the computer as a tool for observing and modulating brain cognition, directly "bombard", "modulate" and "do imaging of" the cognitive activity and content of the brain and build a model of "the brain universe" based on man-machine interaction [5-8]. The bisimulation is other ideas approach to the analysis of Human-Computer Interaction [9]. 


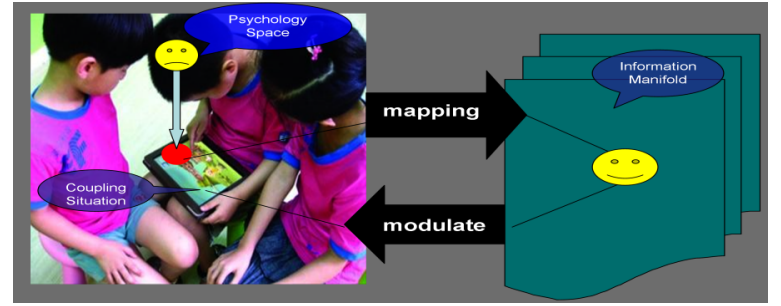

Fig. (1). The level of information to study the cognitive activity of the brain.

\subsection{Brain Imaging of Cognitive Function Based on En- ergy}

The human brain is the dissipation structure of matter, energy and information. In a sense, brain research is about detecting and scanning matter, energy and information in the brain, shooting and collecting data using instruments and finally using these data to understand the brain. Biology and anatomy mainly focus on studying the physical structure of the brain, while EEG (electroencephalogram) and ERP (event-related-potential) focus on studying the brain from the perspective of energy and they are the hottest areas of the current brain research. MIT is even "lightening" the brain. While conducting in-depth study of matter and energy, we shouldn't forget that the core function of the brain is to process symbols and information. The brain is the result of information dissipation of individuals over a number of years and of biological systems over a hundred million years. We should not simply study brain cognition from the perspectives of matter and energy, but should expand our study to the brain from the perspectives of information and content.

Study of the information and content of brain cognitive activity is a new paradigm of brain research and we need to innovate the information observation system, the data collection method and the reconstructed cognitive space of the brain. Fig. (1).

The energy level, Brain technology patents primarily to detect relationship of brain energy, behavior, functional. Such as, Examination image of brain cognitive functions, Brain computer interface (BCI) [10-12].

\subsection{The Cognitive Dynamic System}

Observing and analyzing the content of brain cognitive activity and understanding the cognitive logic and law are important work of cognitive psychology. Traditional cognitive psychology research treats the computer as a tool for data analysis rather than a tool for collecting the content of brain cognition. However, in the aspects of building the models of cognitive psychology, Piaget introduced many mathematical concepts into psychological research and did a lot of groundbreaking work [13]. For example, he introduced mathematical theories such as Category, Morphism, Group and Lattice to the formalization of cognitive logic. We transfer the cognitive experiments of traditional cognitive psychologists based on objective reality to the virtual manmachine interaction interface and rely on the compute to realize the continuous and direct observation of cognitive psychology.
In 1995, T.van Gelder gave his dynamic hypothesis that the cognitive system cognizing the world is a dynamic system and the best understanding to understand the cognitive system is from the dynamic perspective [14]. The dynamic hypothesis describes cognition on the basis of mathematical dynamic systems theory and uses mathematical concepts, such as state space, attractor, trajectory and deterministic chaos, to explain the internal cognitive processes of the cognitive subjects interactive with the environment. Differential equations are used to express the cognitive trajectory of the cognitive subject in the state space. In other words, cognition is described as the multi-dimensional space constituted by all the possible ideas and behaviors of the cognitive subject. In particular, cognition is carefully studied based on the ideological trajectory of the cognitive subject in a certain environment and under certain pressure. The ideas and behaviors of the cognitive subject are subject to the differential equation. The variables in the system change constantly. The system is subject to the nonlinear differential equation and is generally complex and certain [15-18].

On the basis of man-machine interaction, we can collect more accurate cognitive information and build controlled digital environment of cognitive dynamics so as to provide a precise laboratory for cognitive dynamics research, such as building cognitive digital manifolds and high-energy physical "cloud chambers" and "accelerators" to explore cognitive secrets $[7,8]$.

The cognitive dynamic system focused on the content, and related patents also appeared, such as Cognitive function testing system, semantic cognitive map $[12,19]$, however, the content are the result of the behavior, rather than direct observation of brain activity, cognitive process.

\section{BSM COGNITIVE MANIFOLDS BASED ON MAN- MACHINE INTERACTION}

On the basis of the functional simulation of man-machine separation, we can know that brain research has such a research mode as the structural observation of man-machine fusion according to the principle of complementarity philosophy, that is to say, using observation tools directly collect data about the brain cognitive process. Is this mode existent and possible? Because we cannot enter the brain, a bold idea is to induce cognition inside the brain into the environment outside the brain for observation. Will cognition occur outside the brain? We should study man-machine interaction in the leading field of cognitive philosophy so as to seek the possibility.

\subsection{The Perspective of Cognition Outside the Brain}

Does cognition only occur inside the neurocranium? Or is the neurocranium the moving boundary of brain cognition? The occurrence, movement and evolution mechanisms of cognition are hotspots of cognitive philosophy[20-23].

1. Situated Cognition: The development of cognition, knowledge and intelligence is rooted in the interaction, mutual adjustment and adaptation of the agent and environment. Cognition is an event existing in the overall context of the agent-environment interaction 
and correlation. The environment, more than just being a cognitive object in epistemology, also supports the existence of the agent. Cognition is existenceoriented, activity-directed and it is an activity pointing to the environment and can exist outside the brain.

2. Embodied Cognition: The Organism and the environment enfold and unfold each other in basic cycling, which represents the life itself. Cognition and knowledge are generated in the life system coupled with the environment, which is known as the secondgeneration cognitive scientific perspective and is a development of the former perspective thinking cognition equivalent to computing.

3. Distributed Cognition: Hutchins published his representative work "Cognition in the wild" in 1995 and held that cognitive activity occurs and is distributed in the cognitive environment composed of other humans, technical artifacts, external representation and the environment. Cognition is a distributive phenomenon and it has already been applied to the systematic analysis of man-machine interaction. Jim Hollan and Ed Hutchins established Distributed Cognition [23].

4. Extended Cognition: Extended cognition and extended mind are hypotheses in cognitive philosophy which have caused international shock and controversy. Proposed by Clark in 1998 [14], this hypothesis claimed that mind can extend outward and enter a series of cognitive objects, such as tools, media and other people. The cognitive agent can extend to tools outside the brain. Extended cognition emphasizes that cognition is dynamic coupling. Clark and Chalmers held the view that the human body and the external environment are associated with each other in an interactive way, and they constitute a coupling system, in which all constituents take effects through a positive causality. If these external constituents are removed, the behavior capability of this system will be declined, just like removing the constituent parts of the brain. For this reason, the coupling process should be considered as a cognitive process no matter whether it completely exists in the brain or not [20].

To sum up the edge cutting philosophical concepts of cognition, we think that cognition inside the brain can cross the boundary of the skull to move outside the brain. Technologically speaking, it is feasible for cognition inside the brain to be coupled by the situation or induced into the environment outside the brain in the mode of the agent.

\subsection{Man-Machine Coupling}

In order to explore the law of brain movement at the information and symbol levels, we need to study the brain in the active state of the information processing of the brain, select an instrument and an information processing state of the brain for online correlation and coupling and collect the information in the active process of brain information rather than matter or energy. We call this brain state an information brain (iBrain) so as to distinguish from the research paradigms at the matter and energy levels. In this way, the recorded data will reflect the secrets of brain information processing. For the time being, we won't study the states of matter and energy in brain information processing; instead, we will focus on brain information processing itself. Undoubtedly, the computer is the best choice [7-8].

When an individual operates the computer, the computer software system responds to the relevant operation of the individual, such as recording the cognitive processes and determining the next cognitive operation according to relevant logics so as to reach the information coupling state where the brain and the computer input and output to each other.

Definition 1 Man-Machine Cognitive Coupling State: (1) The researcher designs cognitive content, logic, rules, procedures and orbits; (2) the individual brain understands the cognitive situations designed by the researcher; (3) the individual brain responds to the situations after understanding; (4) the computer determines the next operation node according to the response process of the brain so as to realize cognitive modulation; (5) if the behaviors of the brain act in accordance with the predetermined orbit, the designer's cognitive design matches the individual cognition and the related cognitive law is validated; (6) otherwise, either the cognitive law needs to be modified or the cognitive modulation needs to be improved etc.

The sum of the objects, content, rules, processes, orbits and so on operated by brain cognition is called the cognitive information space which is introduced to "materialize" the information activity of the brain by using the computer software, and using the Agent to replace the brain behavior of the object. Information coupling can be abstracted into the Agent behavior in the cognitive information space, similar to the particles in the electromagnetic field.

\subsection{BSM Coupling Cognitive System}

In general, objective objects exist in certain background space and carriers, and in mathematics, it is called manifolds. The man-machine interactive environment existing in the information space is called the cognitive information manifold and constitutes the "brain cognitive body-coupling situation-information manifold" (BSM) digital cognitive system in which the coupling situation is a link between and a combination of information and cognition.

Definition 2 Brain Cognitive Body: the brain cognitive body taking the skull as the physiological boundary is the physiological carrier of brain cognition as well as the material basis for individual intelligence and the highdimensional cognitive manifold, abbreviated as $\mathbf{B}$.

Definition 3 Coupling Situation: the coupling situation is mainly the space of man-machine situation interface which takes the set of objects that can be perceived, operated and cognized by the brain as the boundary, abbreviated as $\mathbf{S}$.

Definition 4 Information Manifold: the information manifold taking the information system as the main carrier is the space where the cognitive subject namely Agent exists and acts in vitro. The low-dimensional projection manifold of high-dimensional cognitive activity is abbreviated as $\mathbf{M}$. 


\subsection{The Cognitive Coupling Digital Manifold}

We hope to build a visual cognitive digital dynamic system which is similar to the cloud chamber in cosmology study. It is full of clean air and alcohol (or ether) saturated steam. When an invisible charged particle breaks in, it will become the core of the "cloud" condensation and form a cloud point to display the "footprint" of particle movement.

Definition 5 Cognitive Coupling Digital Manifold: Based on the perspective of cognition outside the brain, the man-machine interactive environment of coupling cognition and the Agent technology, cognition is projected onto the information manifold where the cognitive process is imaged. This manifold is called "the cognitive coupling digital manifold" or "the cognitive digital manifold". We hope that the "cognitive digital manifold" can become the "cloud chamber" of cognitive observation.

The brain cognitive body is a high-dimensional cognitive manifold and needs to be projected onto the low-dimensional manifold. The dimensionality reduction of the brain cognitive manifold involves problems, such as low-dimensional manifold structures, vectorization of situation spaces, symbolization of cognitive activity and cognitive dynamic structures. BSM is a tool platform for realizing cognitive digital manifolds.

Assume that a researcher collects the information about the cognitive process in the following ways: 1) photographing: obtaining the visual information about the activities of the cognitive situation; 2) recording: recording the acoustic information about the activities of the cognitive situation; 3) camera shooting: recording the video information in the scene of the cognitive situation. These data are closely coupled and are difficult to be decomposed, merged, retrieved and processed. It is essential to find other ways to innovate the vector technology for the cognitive situation.

BSM information observation is faced with the following problems:

1) Design of the situation for induction: how to design an effective coupling situation to induce cognition inside the brain into the manifold outside the brain;

2) Deployment of situation sensors: deploying a variety of situated cognition sensors to conduct multi-dimensional observation of BSM;

3) Sampling of cognitive information: because there is a lot of information in the space and process of the cognitive situation, it is necessary to scientifically collect highdimensional information so as to facilitate information processing and avoid data "disasters";

4) Characterization of cognitive manifolds: how to characterize complex BSM cognitive manifolds in order to ensure the integrity of cognitive information manifolds;

5) Reconstruction of cognitive situations: involving the selection of low-dimensional manifolds and cognitive logical structures;

6) The dynamic system of cognitive manifolds: describing the dynamic axioms, rules and mathematics of cognitive information manifolds so as to predict and control cognitive behaviors.

\section{THE MATHEMATICAL FOUNDATION OF COG- NITIVE DIGITAL MANIFOLDS OF MAN-MACHINE INTERACTION BASED ON CATEGORY THEORY}

It is necessary to sort out BSM mathematical models based on the BSM brain cognitive observation platform so as to provide fundamental mathematical support for the research into BSM. From a certain perspective, BSM aims at mirroring and homogenizing $\mathbf{B}$ into $\mathbf{M}$ through $\mathbf{S}$. we will analyze the relationship of BSM from the perspective of category theory.

Morphism, category and category theory can be considered as important imagination approach when we human beings cognize tools. It provides a new perspective to visualize the dynamic changes from concreteness to formalization during the cognitive process. The famous psychologist Piaget started the research into category-based cognition from 1960s.

\subsection{BSM Cognitive Category}

In modern mathematics, category is one of the most basic concepts. A category $\mathrm{C}$ consists of the following elements [24]:

$\mathbf{C}_{1}$ : a class ob(C) of objects;

$\mathbf{C}_{2}$ : a class hom $(\mathbf{C})$ of morphisms, or arrows, or maps. Each morphism $f$ has a unique source object $\mathrm{A}$ and a target object $\mathrm{B}$ where $\mathbf{A}$ and $\mathrm{B}$ are in $\operatorname{ob}(\mathbf{C})$. We write $f: \mathbf{A} \rightarrow \mathbf{B}$, and we say " $f$ is a morphism from A to B". We write hom(A, B) or homC $(\mathbf{A}, \mathbf{B})$ to denote the hom-class of all morphisms from $\mathbf{A}$ to $\mathbf{B}$. (Some authors write $\operatorname{Mor}(\mathbf{A}, \mathbf{B})$ or simply $\mathbf{C}(\mathbf{A}$, B) instead).

$\mathbf{C}_{3:}$ a binary operation called composition of morphisms. For every three objects $\mathbf{A}, \mathbf{B}$ and $\mathbf{C}$, hom $(\mathbf{B}, \mathbf{C}) \times$ hom $(\mathbf{A}, \mathbf{B}) \rightarrow \operatorname{hom}(\mathbf{A}, \mathbf{C})$. The composition of two morphisms $\boldsymbol{f}$ : $\mathbf{A} \rightarrow \mathbf{B}$ and $\boldsymbol{g}: \mathbf{B} \rightarrow \mathbf{C}$ is written as $\boldsymbol{g}$ of or $\boldsymbol{g} \boldsymbol{f}$ and meets the following three axioms:

$\mathbf{A}_{1}$ disjointness: unless $\mathbf{A}=\mathbf{A}^{\prime}$ and $\mathbf{B}=\mathbf{B}^{\prime}$, morphism sets $\mathbf{C}(\mathbf{A}, \mathbf{B})$ and $\mathbf{C}\left(\mathbf{A}^{\prime}, \mathbf{B}^{\prime}\right)$ cannot intersect;

$\mathrm{A}_{2}$ associativity: if $f: \mathrm{A} \rightarrow \mathrm{B}, g: \mathrm{B} \rightarrow \mathrm{C}$ and $h: \mathrm{C} \rightarrow \mathrm{D}$, then $h \circ(g \circ f)=(h \circ g) \circ f$;

$\mathbf{A}_{\mathbf{3}}$ identity: for every object $\mathrm{Y}, \operatorname{hom}(\mathbf{Y}, \mathbf{Y})$, there exists a morphism $1_{\mathrm{Y}}$ such that for every morphism $\boldsymbol{f} \in \operatorname{hom}(\mathbf{X}, \mathbf{Y})$, $\boldsymbol{g} \in \operatorname{hom}(\mathbf{Y}, \mathbf{Z})$, we have

$$
1_{\mathrm{Y}} \circ f=f, g \circ 1_{\mathrm{Y}}=g \text {. }
$$

$\mathbf{1}_{\mathbf{Y}}$ is unique and is also called the identical morphism of object $\mathbf{Y}$. If $f \in \operatorname{hom}(\mathbf{X}, \mathbf{Y})$ and $\boldsymbol{g} \in \operatorname{hom}(\mathbf{Y}, \mathbf{X})$, there is $\boldsymbol{g} \circ \boldsymbol{f}=\mathbf{1}_{\mathbf{X}}$, and then morphism $g$ is called the left inverse of $\boldsymbol{f}$ and $\boldsymbol{f}$ is called the right inverse of $\boldsymbol{g}$. The morphism which is both the left inverse and the right inverse of $\boldsymbol{f}$ is called the two-sided inverse of $\boldsymbol{f}$.

The morphisms in a category are sometimes called "arrows" and this name originates from commutative diagrams.

Definition 6 Cognitive Category: The structures and relations that exist in the brain cognitive body and the artificial intelligent body, are abstracted into cognitive objects, cognitive morphisms and cognitive structures. They are called 


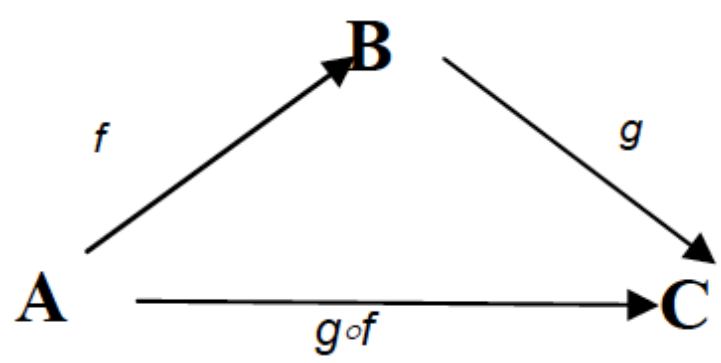

Fig. (2). Commutative diagram for the cognitive morphism.

cognitive categories when described using categories of mathematical methods. Fig. (2).

Definition 7 Cognitive Morphism: in the cognitive category $\mathbf{C}$, (1) the objects and phenomena that can be cognized constitute a cognitive class and the object $\operatorname{ob}(\mathbf{C})$, $\mathbf{A}, \mathbf{B} \in \mathrm{ob}(\mathbf{C})$; (2) whether based on knowledge connection, cognitive logic, experience or imagination, cognitive operation that establishes the relationship between $\mathrm{A}$ and $\mathrm{B}$ is a cognitive morphism $f: \mathbf{A} \rightarrow \mathbf{B}$, hom(A, B). Undoubtedly, $\mathbf{A}, \mathbf{B}, \mathbf{C} \in \operatorname{ob}(\mathbf{C})$ form the cognitive morphism chain $\operatorname{hom}(\mathbf{B}, \mathbf{C}) \times \operatorname{hom}(\mathbf{A}, \mathbf{B}) \rightarrow \operatorname{hom}(\mathbf{A}, \mathbf{C})$ and meet transitivity, etc.; (3) an identical cognitive morphism is determined operation of cognitive objects. Morphisms are similar to cognitive operation and cognitive operators. The correlation between cognitive objects can also be expressed by using arrows.

Definition 8 BSM Category System: in the manmachine interaction system, we assume that the brain cognitive body is category $\mathrm{B}$, the coupling situation is category $\mathrm{S}$, the information manifold is category $\mathrm{M}$ and all of them are called category BSM or man-machine coupling category. Then the following morphisms exist in category BSM:

1) Cognitive Morphism: the morphism existing between the cognitive objects inside the brain is based on brain thinking and connects the cognitive space inside the brain.

2) Symbol Morphism: the morphism existing between manifold objects outside the brain. is based on symbol computation of the computer and it is the morphism in the system of symbols, logic and operation.

3) Coupling Morphism: This morphism is constructed between the cognitive morphism and the symbol morphism by the coupling situation, so as to build the mutual links between these two parts.

The cognitive category and the category BSM system are built to provide a mathematical basis for the observation, reconstruction and dynamic research of cognitive structures.

\subsection{Cognitive Natural Isomorphism and Cognitive Cou- pling Transformation Theorem}

BSM has become a platform for observing the cognitive structure inside the brain. There are many problems about scientifically collecting data in the man-machine interactive environment. For example, is it possible to observe the cognitive structure inside the brain in the man-machine interactive environment? Is the cognitive object observed in the man-machine interactive environment consistent with that

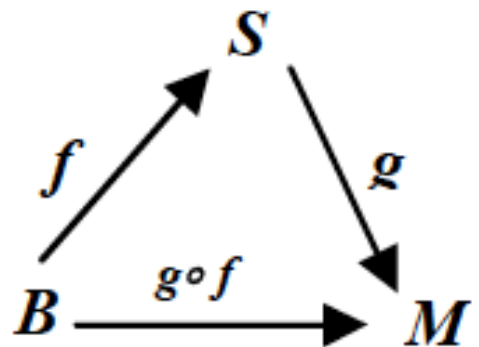

Fig. (3). Cognitive Natural Isomorphism.

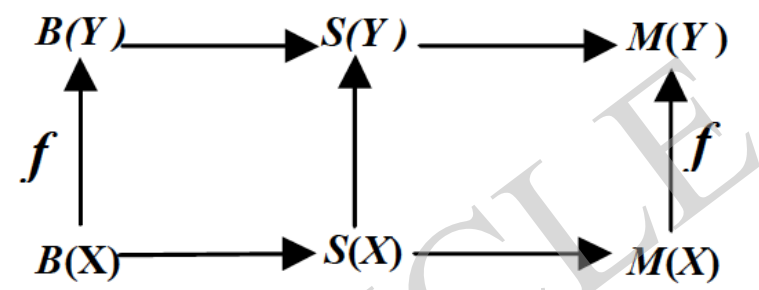

Fig. (4). Cognitive Natural Transformation.

inside the brain? Is the cognitive morphism observed in the man-machine interactive environment consistent with that inside the brain? On the basis of hypotheses about the category and the cognitive category, we will obtain the following two theorems:

Theorem 1 Information - Cognitive Space Cognitive Natural Isomorphism: The morphism $\boldsymbol{f} \in \operatorname{hom}(\mathbf{X}, \mathbf{Y})$ is called equivalent $(f: X \approx Y)$. If the two-sided inverse $f$ ${ }^{1}$ hom $(\mathbf{Y}, \mathbf{X})$ exists, i.e. there is an equivalent morphism between information and cognitive category, then the objects of the cognitive category are equivalent. This is called the Cognitive Natural Isomorphism Theorem, as shown in Fig. (4).

$$
\begin{aligned}
& \operatorname{hom}(\mathbf{B}, \mathbf{S}) \times \operatorname{hom}(\mathbf{S}, \mathbf{M}) \rightarrow \operatorname{hom}(\mathbf{B}, \mathbf{M}) \\
& \operatorname{hom}(\mathbf{M}, \mathbf{S}) \times \operatorname{hom}(\mathbf{S}, \mathbf{B}) \rightarrow \operatorname{hom}(\mathbf{M}, \mathbf{B})
\end{aligned}
$$

Theorem 2 Information - Cognitive Space Cognitive Coupling Transformation: According to the Category Functor Principle, the cognitive functor keeps the coupling transformation between BSM and the covariance of the cognitive law between the cognitive morphism and the information manifold morphism inside the brain, as shown in Figs. $(\mathbf{3}, \mathbf{4})$.

The cognitive natural isomorphism theorem and the cognitive coupling transformation theorem guarantee the unity of the cognitive objects in the cognition - information space and the covariance of the cognitive law, serving as a mathematical basis for BSM cognitive observation and imaging.

\section{VISUAL AND COMPLEX COGNITIVE NET- WORKS}

\subsection{High-Dimensional Cognitive Manifolds and Complex Network Homomorphism}

In mathematics, a manifold can be approximated using a graph and homogenized at a certain angle. Graph $\mathrm{G}=(\mathrm{V}, \mathrm{E})$ contains two sets: the vertex set $\mathrm{V}$ and the edge set $\mathrm{E}$. For the 


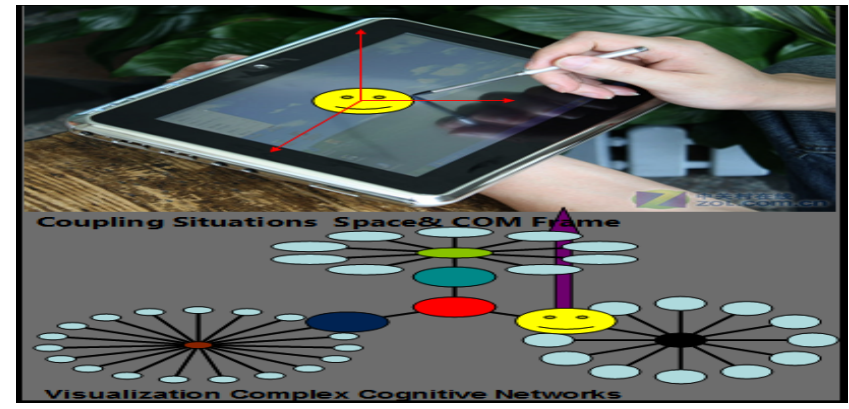

Fig. (5). The situation is the local observation and measurement of the cognitive manifold.

sample data set $\mathrm{x}$ sampled from a d-dimensional manifold, the first step is to establish one-to-one correspondences between data points and the vertexes of Graph $G$ and define the similarity between two data points as the edge. In this way, a corresponding graph is established according to data points. A graph and a manifold have many similar properties. The most important one is that both can be inserted into the Euclid space. For a manifold, a corresponding graph is a topological object and its topological property is expressed by the weight. We use complex cognitive networks [25-30] to describe cognitive manifolds where network nodes represent cognitive objects and sides represent the logical relationship between cognitive points.

Currently, the complex network has been a research hotspot which is not only the framework for describing various open and complex systems from technology, biology to society, but also a powerful tool for studying their topologies and dynamic properties. In the cognitive field, the schema in the system of cognitive psychology, the knowledge graph, the concept map and the thinking navigation map in knowledge engineering represented by Piaget are associated with the concept of complex networks. Fig. (5).

Therefore, on the basis of the cognitive coupling observation and the cognitive imaging inside the brain, we can use complex networks to homogenize the cognitive structure inside the brain and reduce the dimensionality of the highdimensional cognitive manifold so as to visualize cognition inside the brain.

\subsection{Cognitive Symbol Sequence}

In scientific research, precise measurement will surely bring about a lot of data, while the characteristic quantities used to describe the fundamental natures of things are rare. In order to obtain those few characteristic quantities, it is not necessary to proceed from a great number of precise original data. Actually, the entire system of natural sciences is a "coarse-grained" or "reduced" description of things. This platform can be well implemented in the study of dynamic systems. Symbol dynamics is a method for strictly describing dynamical processes in finite precision [31].

We establish cognitive operation to describe symbol sets and conduct "reduced" description of the cognitive processes in the BSM situation so as to solve "data explosion" problems in the collection of situation data.
BSM is a complex system, so it is necessary to conduct multi-dimensional observation and collect information about its activities. The use of cognitive symbol sequences in describing cognitive processes at the symbol and logic levels also meets the trends in the mathematical systems of current complex systems, namely the synthesis, generalization and self-repairing of value and symbol operations. We construct parallel processing and Agent calculation model. For example, the adaptability basic mechanism "stimulus-response" model in the CAS [32] model is expressed by using the "IF-THEN" rule.

In the BSM coupling situation, it is necessary to conduct "coarse-grained, reduced" processing of cognitive interaction scientifically. For example, when a student dictates the word BOOK, he first knocks B, then $\mathrm{U}$ and then $\mathrm{K}$. After realizing his mistakes, he backspaces and knocks two Os. This process can be expressed with the following three sequences: B-BUBUK-BOK-BOOK, B-U-K-O-O and 1 (1 stands for correctness and 0 stands for wrongness). Obviously, the first one can best reflect the cognitive process of the student and the third one is a simple result containing the least information. Representation of a question by using $\mathrm{Q}$ and representation of a choice question by using $\mathrm{S}$ are embodiments of symbolization.

Definition 9 Cognitive Symbol Set: The symbol $\mathrm{S}=\{\mathrm{a} \in \mathrm{S} \mid \mathrm{S}$ is the character of cognitive significance that can be stored, transmitted and processed by the computer $\}$ and is also called cognitive symbol.. Cognitive symbols can express cognitive elements, such as cognitive time, space, processes and logic.

Definition 10 Cognitive Operation: let $S$ be the cognitive symbol set. The cognitive operation $\varphi:: \mathrm{S} \otimes S \rightarrow \mathrm{S}$ is called a binary cognitive operation in $\mathrm{S}$, a cognitive operation for short. $\otimes$ stands for "cognitive operator". The cognitive operation $\varphi$, the morphism, the map and the function have similar meanings. Cognitive coupling is the bijection between two cognitive systems.

Cognitive operation is a core concept. Apart from being expressed with mathematical operators in algebra, such as plus, minus, matrix, cognitive operation can also be described using mathematical logic and formal languages, such as IF-THEN statements and codes for computer programming languages.

Definition 11 Cognitive Symbol Sequence: number or symbol sequences that have cognitive operation connotation and can reflect the process of cognitive operation are called cognitive symbol sequences. That is, the cognitive process $\mathrm{P}$ is expressed using the elements $\mathbf{S 1}, \mathbf{S 2}, \ldots, \mathbf{S x}-\mathbf{1}, \mathbf{S x}$ in $\mathrm{S}$ to constitute a cognitive symbol sequence, CSS for short [33].

Definition 12 Cognitive Logic: cognitive logic is a CSSbased logical system oriented to the reasoning law of cognitive processes. Logical laws are expressed by using a variety of languages including computer languages. In terms of forms of expression, the L-system, the IFS system and the cognitive symbol sequence in the fractal field have many similarities. 


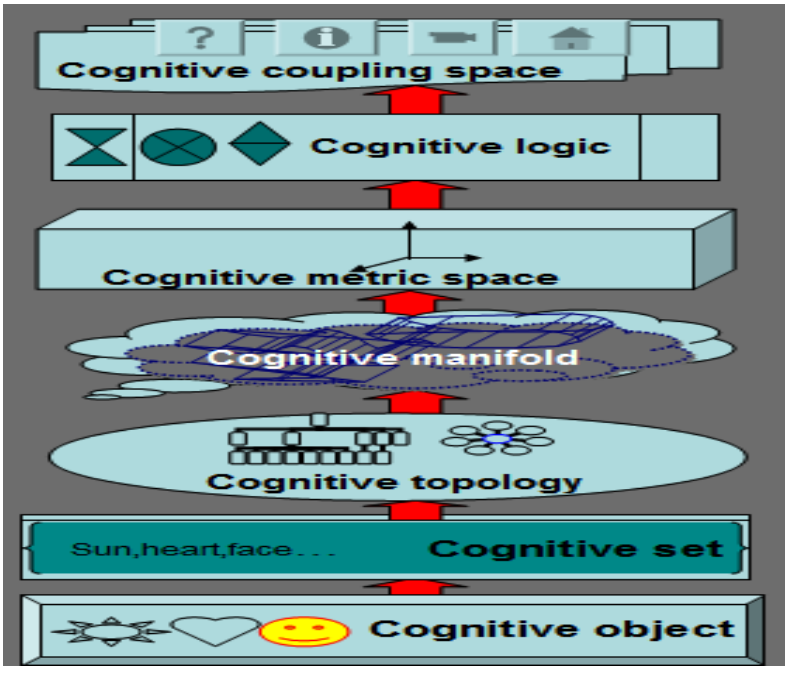

Fig. (6). BSM mathematical architecture.

\section{THE DYNAMIC SYSTEM ARCHITECTURE OF THE COGNITIVE DIGITAL MANIFOLD}

In BSM, cognition is a high-dimensional manifold. It is difficult to construct a coordinate system of the entire manifold and it is only possible to observe the parts of the manifold. Consistent with the situation information flow theory "one situation is a finite part or fragment of the world", we think that the situation interface is the local measurement of the cognitive manifold. We 1) utilize coupling situations to observe cognitive manifolds, 2) collect data; 3) homogenize cognitive manifolds into complex cognitive networks and 4) make cognitive manifolds symbolic and mathematical.

The BSM manifold is structured as follows:

1. Cognitive object: objects deployed in man-machine interactive equipment that can be cognized by sensors.

2. Cognitive set: the sum of the cognitive objects constituted according to certain purposes, processes and functions in a certain situation or process.

3. Cognitive topology: cognitive topologies are constructed on the basis of cognitive sets and topology axioms.

4. Cognitive manifold: cognitive manifolds are formed on the basis of cognitive topologies and additional metric structures, such as mathematics, weights, distances, flows and logic.

5. Cognitive measurement: cognitive situations are used to couple cognition inside the brain in order to measure cognition.

6. Cognitive logic: restrictive relations are established between situations at the level of cognitive logic so as to construct cognitive logic bodies.

7. Cognitive coupling: situations and brain cognition interact and collect information about activities inside the brain.

8. A visual and complex cognitive network covers all of the above descriptions and is the expression and realization of the above structures [34], Fig. (6).

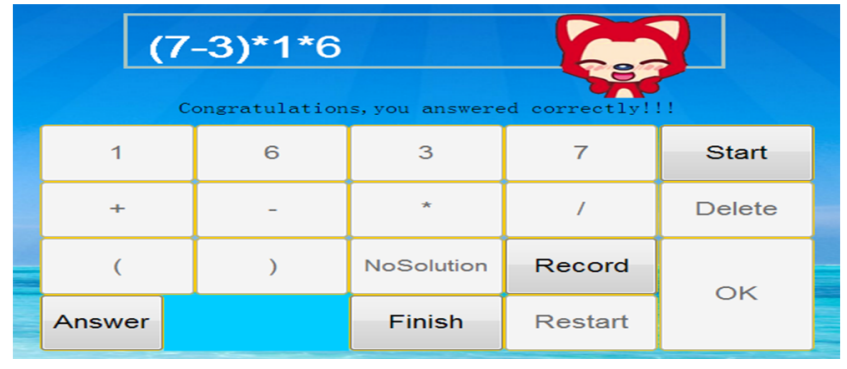

Fig. (7). The man-machine interface for cognitive symbol sequence collection for the 24-point calculation.

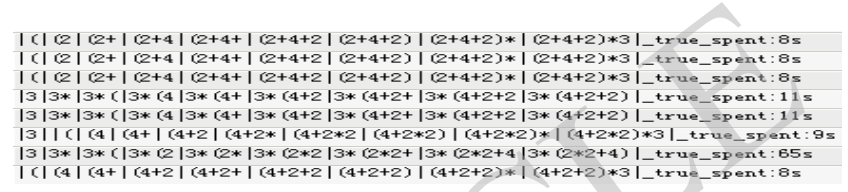

Fig. (8). The cognitive symbol sequence for the 24-point calculation.

Manifolds, state space or phase space, paths or trajectories, attractors, stability, coupling, bifurcation, deterministic chaos and sensitivity to initial condition, etc. are important concepts of cognitive dynamic systems. BSM can collect cognitive manifold information through time parameters and reconstruct metric spaces for the construction of cognitive dynamics.

Base on the coupling principle, BSM observe brain cognitive activity data with a non-contact methods. Non contact method can be conveniently applied in the aspects of brain advanced cognition, such as patent "Cognitive function within a human brain" [35].

\section{APPLICATIONS BASED ON COGNITIVE DIGITAL MANIFOLDS}

\subsection{Coupling Imaging of Cognitive Structures}

Take the 24-point cognitive computing for students as the example to conduct the above analysis. In the 24-point thinking game, students are given 4 numbers from 1 to 9 and then combines them with four math operations such as plus, minus, times or division, then the result is 24 (or no solution).

Using the 24-point cognitive computing man-machine interaction software system, where each calculation is a record, nearly 80,000 cognitive data records are collected from more than 2000 students ranging from third-grade pupils to college students and then imaging of cognitive structures are done based on the above approach.

1) The Interface for Cognitive Symbol Sequence Collection: The computer gives 4 numbers randomly and then the user clicks on corresponding symbols to form an arithmetic expression, shown as the cognitive symbol sequence man-machine interface for the 24-point calculation in Fig. (7).

2) Cognitive Symbol Sequence: The related data is collected when the learner clicks on the related cognitive sensors and the records are generated, shown in Fig. (8) (including data such as the user ID and time). 


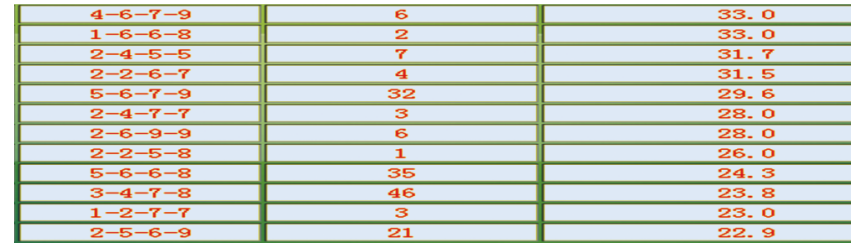

Fig. (9). The average time for the 24-point game.

\begin{tabular}{|l|l|l|l|}
\hline$(A+B+C)^{*} D$ & $\left((A+B)^{*} C\right) / D$ & $\left(A^{*}(B+C)\right)^{*} D$ & $A^{*}((B+C)+D)$ \\
\hline$A^{*}((B+C)+D)$ & $(A+B)^{*}(C / D)$ & $A^{*}\left((B+C)^{*} D\right)$ & $A^{*}(B+(C+D))$ \\
\hline$A^{*}(B+(C+D))$ & $(A+B)^{*} C / D$ & $(A * B+C)^{*} D$ & $A^{*}(B+C+D)$ \\
\hline$((A+B)+C)^{*} D$ & $A^{*}\left(B^{*}(C+D)\right)$ & $A^{*}(B+C)^{*} D$ & $A^{*}((B+C)+D)$ \\
\hline$(A+(B+C))^{*} D$ & $\left(A^{*} B\right)^{*}(C+D)$ & $((A / B)+C)^{*} D$ & $A^{*}(B+(C+D))$ \\
\hline$(A+B+C)^{*} D$ & $A^{*} B^{*}(C+D)$ & $(A / B+C)^{*} D$ & $A^{*}(B+C+D)$ \\
\hline$\left((A+B)^{*} C\right) * D$ & $A^{*}\left(B^{*}(C+D)\right)$ & $((A+B)+C)^{*} D$ & $\left(A^{*}(B+C)\right)^{*} D$ \\
\hline
\end{tabular}

Fig. (10). Part of the patterns.

3) Cognitive Set: How to handle large quantities of cognitive symbol sequences? Classifying and setting up sets are fundamental work. For example, when the records are classified according to the average time for each calculation, then the records with the toughest solution can be searched. In Fig. (9), the calculation questions, the test count and the average time are all listed. For example, the $3-3-5-8$ is tested 81 times and the average time is 15.1 seconds.

4) The Analysis of Cognitive Topology: The cognitive topology space is built based on the cognitive set. For example, using the algebra approach, $(4+2+2) * 3$ can be topologized into $(\mathrm{A}+\mathrm{B}+\mathrm{C}) * \mathrm{D}$, therefore cognitive records like $(3+3+2) * 3$ and $(1+3+2) * 4$ can all be mapped into the image $(\mathrm{A}+\mathrm{B}+\mathrm{C})^{*} \mathrm{D}$, and all the images can compose the new level cognitive topological space, which is also called quotient topological space.

After the algebraization analysis of 62401 records, 232 images are obtained, which is much less than the number generated purely by permutation and combination, which shows that cognitive operation is different from the pure mathematical way. Because these cores are fundamental, we call them the gene of 24-point cognitive computation, as shown in Fig. (10).

Cognitive Topological Imaging: Four sides of a quadrangle Q1, Q2, Q3 and Q4 are constructed to represent the values of four numbers in the 24-point game, with each cognitive calculation of the 24-point game mapped to a point on the quadrangle. Then the $\mathrm{Q}$ coordinates can form the diagram shown in Fig. (6). Furthermore, different colors can be given to the points to represent the corresponding time level of the cognitive calculation. For example, red means spending the longest time, while green means spending the least time. Then a cognitive topology diagram is formed, shown as Fig. (11).

To sum up, the 24-point cognitive calculation is analyzed in details at its process level, topology level and dynamics level based on man-machine interaction. By using the visualization technology, the related abstract data are presented to support educational applications.

\subsection{Observation of Cognitive Motivation Processes}

Man-machine interaction software systems are designed based on specific learning processes to observe learning processes. Generally, the observation procedures are listed as follows [36]:

1) Learning situation interface in man-machine interaction for mental arithmetic: the learning situation in manmachine interaction is designed to learn to observe targets and the learning process will be finished in this interface. Man-machine interactive areas are deployed in the learning situation according to requirements to collect information about man-machine operation.

For example, the mental arithmetic learning system is designed like this: on the left is oral questions and on the right is the numeric keyboard. Rain drops fall continuously before hitting the ground. The answer to this question should be given by using the numeric keyboard and a solution is given by pressing the "Enter" button. If the number inside the input box is not the "rain drop" result shown in the left situation, it will be counted as a wrong operation, and the "rain drops" still exist and keep moving downward. Otherwise, it will be counted as a success and "rain drops" will disappear automatically. The remaining time and scores are shown at the top right corner. Fig. (12).

2) Information collection for mental arithmetic training: collecting information about learning processes based on the content of learning. 10 points will be gained for a correct operation and 5 points will be deducted for a wrong one. The overall score will be calculated by 5 minutes as a training unit.

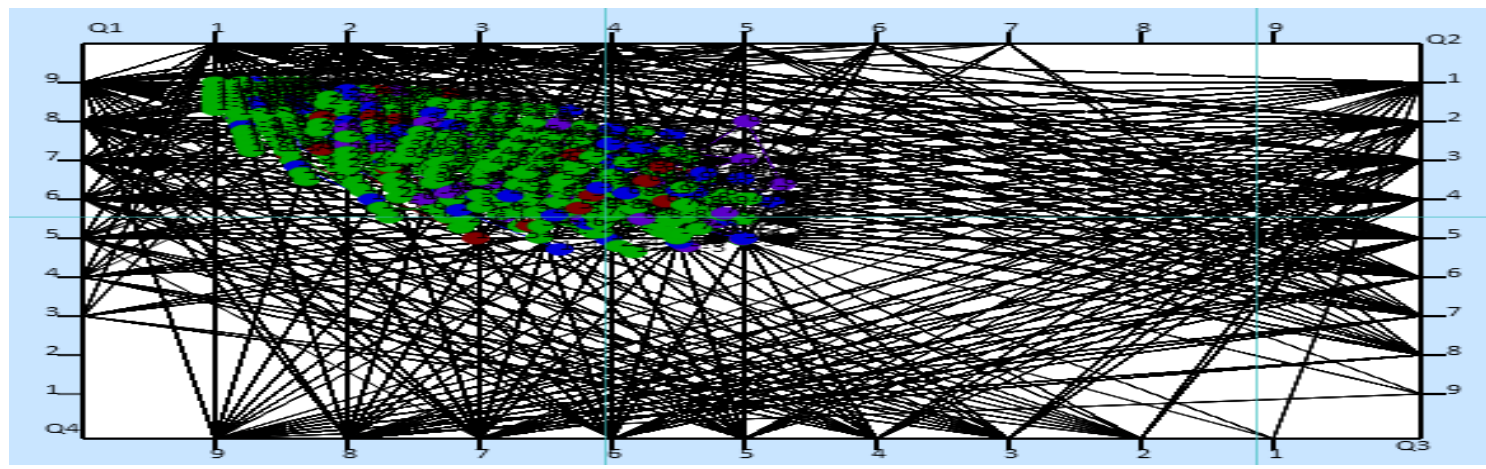

Fig. (11). The imaging of the cognitive topology. 


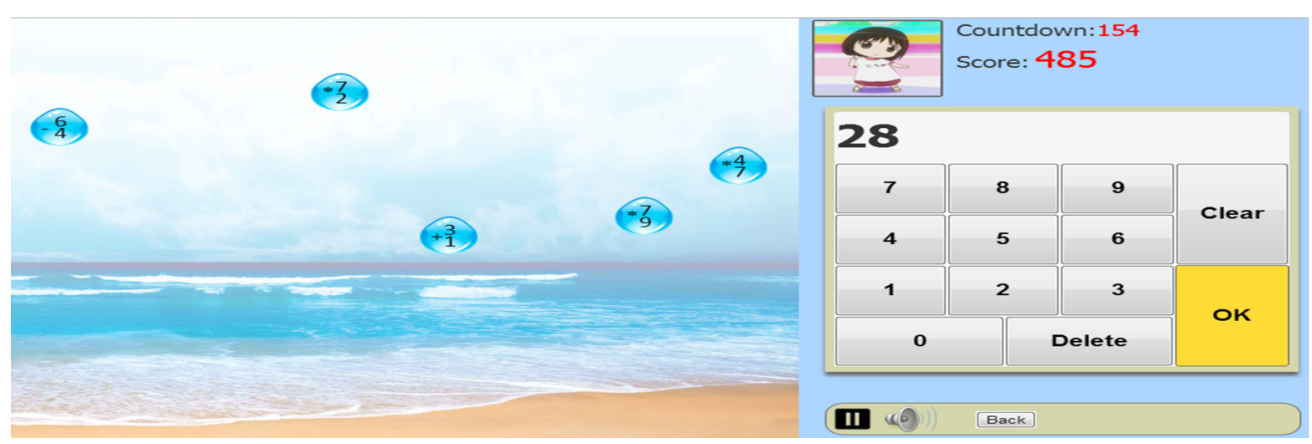

Fig. (12). Man-machine interface for information collection in mental arithmetic training.

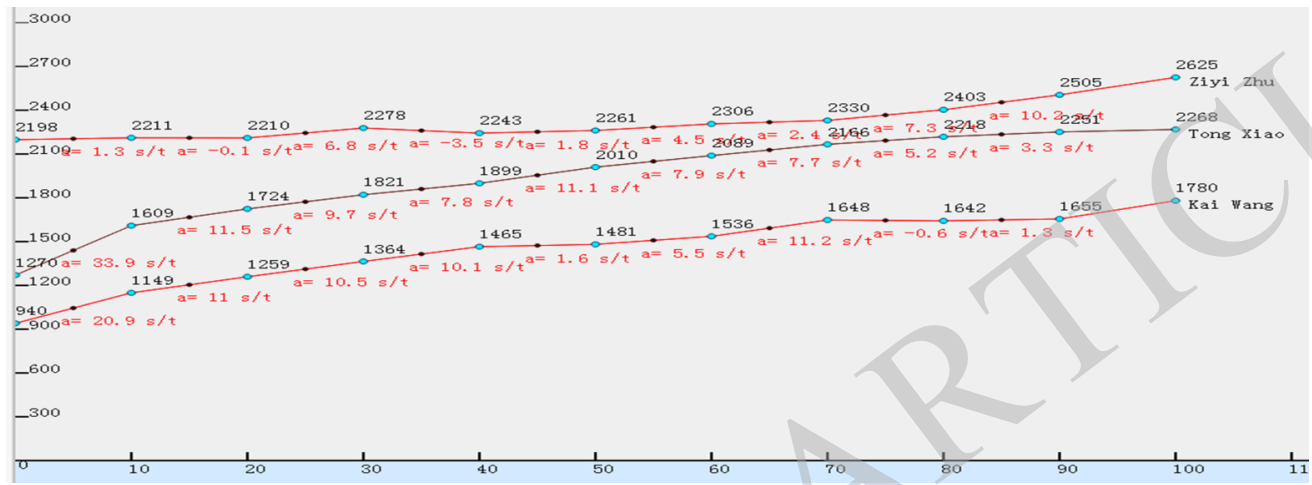

Fig. (13). Speed curves of mental arithmetic training.

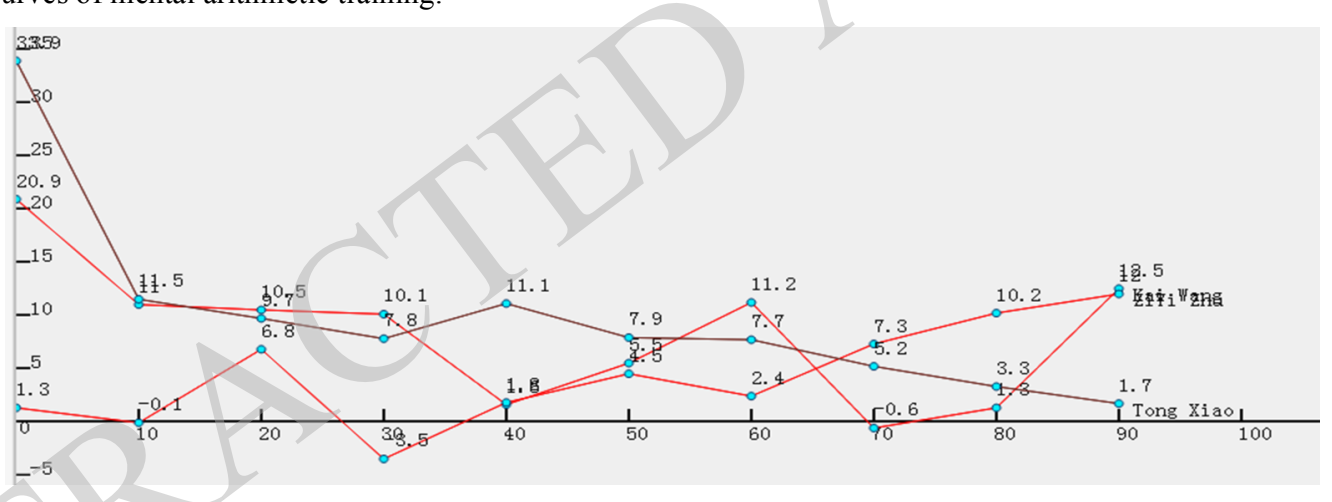

Fig. (14). The acceleration curves for mental arithmetic training.

3) Multi-dimensional variable analysis of mental arithmetic: creating sets is the foundation of mathematic research as well as a vital step for processing cognitive data. The set of multi-dimensional learning objects is built based on individuals, groups, genders, locations, etc. and the multiscale learning set can be created based on learning time and duration, learners' ages, grades and areas.

4) Dynamic analysis of mental arithmetic learning: a coordinate description for mental arithmetic training is established with the training times as the $\mathrm{x}$ axis and the score result of the 5 minute training as the y axis which is called "the progress curve of mental arithmetic", similar to the physical speed in the mechanical system. Fig. (13).

We can get the average speed within certain times according to the formula below, where $\mathrm{Y}$ is the score for mental arithmetic, $m$ and $n$ are times on the $\mathrm{X}$ axis and $\mathrm{V}$ is the average mental arithmetic speed during the $m$ and $n$ training period:

$$
V=\frac{Y_{m}-Y_{n}}{m-n}
$$

To more accurately understand students' progress made in mental arithmetic learning, we create a concept similar to acceleration in physics.

$$
a=\frac{Y_{m}-Y_{n}}{m-n}
$$

In this equation, a represents the progress acceleration of mental arithmetic, $Y$ stands for the training time of $m$ and $n$ on the $\mathrm{X}$ axis. According to the above formula, the learning acceleration of the three students shown in Fig. (14) can be described in the figure below, where $\mathbf{m}-\mathbf{n}=\mathbf{1}$.

When analyzing acceleration curves, we find that all curves have their lowest point, representing the slowest progress even a negative increase, namely regress. The time for 
reaching the lowest point and the acceleration value at the lowest point show the student's personality, denoted by $\mathrm{a}_{\min }$. The times of training performed to reach $\mathrm{a}_{\min }$ are represented by $\mathrm{Ta}_{\min }$. Similar to traditional psychological research, multi increases or negative increases could possibly happen to students. For instance, Fig. (14) shows $\mathrm{a}_{\min }$ and corresponding $\mathrm{Ta}_{\min }$ of three students:

$$
\begin{aligned}
& \mathrm{a}_{\min }=-3.5,-0.6,+2.4 \\
& \mathrm{Ta}_{\min }=40, \quad 80,100
\end{aligned}
$$

Apparently, BSM observe more cognitive process rather than results. Such as patent "Teaching system for improving information retention base on brain-state monitoring, Remote monitoring of patient cognitive function using implanted crm devices and a patient management system" [37$38]$. Based on the cognitive process, we will obtain the cognitive structure, dynamic characteristics.

\section{CONCLUSION}

According to the new cognitive perspective, manmachine systems are integrated systems of information and cognition. Cognition inside the brain is distributed in the environment outside the brain in the form of the Agent. Man-machine interaction is designed to be "the brain cognitive body-coupling situation-information manifold", namely the platform for observing cognitive activities and structures.

From the perspective of the category theory, cognitive morphisms exist in the information-cognition space. The cognitive natural isomorphism and cognitive coupling transformation theorems of the information-cognitive space ensure that the cognitive structure inside the brain is homogenized into the information manifold outside the brain. The covariance between the cognitive orbits of cognitive morphisms, activities and information manifolds make cognitive modulation possible.

The observation of cognitive structures based on BSM provides new research paradigms for the imaging of cognitive structures inside the brain and the observation of cognitive dynamics.

Based on the coupling principles, Non contact cognitive activity information observation will be the next generation of brain technology and patent application hot spot.

\section{CONFLICT OF INTEREST}

The authors confirm that this article content has no conflict of interest.

\section{ACKNOWLEDGEMENTS}

The paper is supported by the following funds: the Education Ministry Fund in Fujian Province (JA12321, JA13318), The Special Fund for Industry Development and the Key Scientific Project Fund in Nanping, Fujian (N2012Z06-7).

\section{REFERENCES}

[1] T. J. Cacioppo, W. V. Hippel, and J.M. Ernst, "Mapping cognitive structures and processes through verbal content: the thought-listing technique", Journal of Consulting and Clinical Psychology, vol. 65 , no. 6, pp. 928-940, 1997.

[2] Y. B. Tal," When the need for cognitive structure does not cause heuristic thinking: the moderating effect of the perceived ability to achieve cognitive structure", Psychology, vol. 1, pp. 96-105, 2010.

[3] D. M. Dimitrov and E. Raykov."Validation of cognitive structures: a structural equation modelinga" Multivariate Behavioral Research, vol. 38, no. 1, pp. 1-23, 2003.

[4] E. Schrodinger, "What is life? hunan science and technology press", vol. 9 , no. $156,2003$.

[5] T. Froese, K. Suzuki, Y.Ogai, and T. Ikegami.'Using humancomputer interfaces to investigate 'mind-as-it-could-be' from the first-personp, "Cognitive Computer, vol. 4, pp. 365-382, 2012.

[6] S. Kim, and S. Kwon, "A perceptual visual feature extraction method achieved by imitating V1 and V4 of the human visual system”. Cognitive Computer, vol. 4, pp. 382-388, 2012.

[7] M. Shimin, and L. Changyong, "Cognitive digital manifold based on CHI," Computer and Information technology, vol. 49, no. 1, pp. 1-8, 2013.

[8] M. Simon, and C. Rengui, "Cognitive coupling states based on tree cognitive fields, "International Conference on Computer Communication and Management Proc. of CSIT, vol.5 , pp.593-597, 2011.

[9] S. Combéfis, and C. Pecheur, "A bisimulation-based approach to the analysis of human-computer interaction," Pittsburgh, Pennsylvania, USA, 2009.

[10] B. Chance, "Examination and imaging of brain cognitive functions", U.S. Patent US 2004/0082862 A1, Apr. 29, 2004.

[11] C. Eric G.W. Schalk, D. W. Moran, J.R. Wolpa, J.G. Ojemann," Computer and Information technology, vol. 49, no.1, pp. 1-8, 2012.

[12] A. Yasumura, and M. Inagaki," Cognitive function testing system, cognitive function estimation system, cognitive function testing method, and cognitive function estimation method", Japanese Patent, vol. 1, 2013.

[13] J. Piyajie, "Morphism and category: comparison and transformation," East China Normal University Press, pp. 11-37, 2005.

[14] V. Gelder, and T.J. "Dynamic approaches to cognition," The MIT Encyclopedia of cognitive sciences, pp. 244-246.

[15] R.F. Port, and T. V. Gelder, "Mind as motion: explorations in the dynamics of cognition," MIT Press, 1995.

[16] R. M. French, and E. Thomas, "The dynamical hypothesis in cognitive science: a review essay of mind as motion," 2001.

[17] E. Thelen, and L. B. Smith, "Dynamic systems theories. Handbook of child psychology," pp. 258-312, 2006.

[18] C. A. Skarda, and W. J. Freeman, " How brains make chaos in order to make sense of the world", Behavioral and brain sciences, vol.10, no. 2, pp. 161-195,1987.

[19] G. A. Ascoli, A. samsonovich, "Semantic cognitive map", U.S. Patent US 8,190,422 B2, May 29, 2012.

[20] A. Clark. "How brains make chaos in order to make sense of the world", Behavioral and brain sciences, vol.10, no. 2, pp. 161-195, 1987.

[21] J. Hollan, E. Hutchings, and D. Kirsh, "Distributed cognition: toward a new foundation for human-computer interaction research", ACM Transactions on Computer-Human Interaction, vol. 7, no. 2, pp. 174-196, 2000.

[22] Y. Xiaohan. "The systematic study on the cognitions-from the perspective of distributed cognition," The Faculty of Humanities, 2010.

[23] X. Yiaohan. "The systematic study on the cognitions-from the perspective of distributed cognition," The Faculty of Humanities, 2010.

[24] B. K. Kylar, and M. N. Jones, "Visualizing multiple word similarity measures, Behavioural Research, vol. 44, 656-674, 2012. M. Shimin, "Visualization complex cognitive networks,"2012 $3^{\text {rd }}$ International Conference on e-Education, e-Business, eManagement and e-Learning, vol. 27, pp. 1-5, 2012. 
[26] M. Shimin, "Complex word network model based on cognitive coupling states," Information Society (i-Society), 2012

[27] O. Sporns, "Network Analysis, Complexity, and Brain Function . Complexity,"Computional Coginitive Neuroscience, vol. 8, pp. 5660, 2002.

[28] W. Zheng, and B. Hao, "Appliction symbol dynamic, shanghai: shanghai scientific and technological education publishing house, pp. 1, 1994.

[29] J.H. Holland, "How adaptation builds complexity, Shanghai Science \& Technological Education Publishing House, 2002.

[30] L. Changyong, and M. Shimin, "Information Sequence of Cognitive-oriented 1:1 Digital Classroom", Journal of Changchun Teachers College, vol. 31, no. 12, pp. 121-124, 2012.

[31] M. Shimin, C. Rengui, and L. Yonglin, "Cognitive topology mapping based on human - computer interaction," Journal of Internet Technology and Secured Transactions (JITST), vol. 1, no. 3, 2012.
[32] A. Lozano, "Cognitive function within a human brain", Journal of Internet Technology and Secured Transactions (JITST), vol. 44, pp. 656-674, 2012.

[33] Y. Sheng, and G. L. Y. Wensen, "Learning dynamics analysis based on computer-human interaction", Journal of Emerging Technologies in Learning, vol. 8, no. 5, pp. 32-36, 2013.

[34] B. Mutlu, and D. J. Szafir, "Teaching system for improving information retention base on brain-state monitoring", Journal of Emerging Technologies in Learning, vol. 44, pp. 656-674, 2010.

[35] R. Wariar, and R. J. Sweeney. "Remote monitoring of patient cognitive function using implanted crm devices and a patient management system", Journal of Internet Technology and Secured Transactions (JITST), vol. 44, pp. 656-674, 2012.

Received: July 23, 2014

Revised: August 12, 2014

Accepted: August 12,2014

(C) Rengui et al.; Licensee Bentham Open.

This is an open access article licensed under the terms of the Creative Commons Attribution Non-Commercial License (http://creativecommons.org/licenses/by-nc/3.0/) which permits unrestricted, non-commercial use, distribution and reproduction in any medium, provided the work is properly cited. 